\title{
Detection of epidermal growth factor receptor mutations in exhaled breath condensate using droplet digital polymerase chain reaction
}

\author{
KAZUYA NISHII $^{1}$, KADOAKI OHASHI ${ }^{2}$, TOMOKI TAMURA ${ }^{1}$, KIICHIRO NINOMIYA $^{1}$, \\ TAKEHIRO MATSUBARA ${ }^{3}$, SATORU SENOO ${ }^{1}$, HIROHISA KANO $^{1}$, HIROMI WATANABE $^{1}$, \\ NAOHIRO ODA ${ }^{1}$, GO MAKIMOTO $^{1}$, HISAO HIGO ${ }^{1}$, YUKA KATO ${ }^{4}$, TAKASHI NINOMIYA ${ }^{2}$, \\ TOSHIO KUBO ${ }^{5}$, HIROMASA YAMAMOTO ${ }^{6}$, SHUTA TOMIDA ${ }^{3}$, KATSUYUKI HOTTA ${ }^{4}$, \\ MASAHIRO TABATA $^{5}$, SHINICHI TOYOOKA ${ }^{6}$, YOSHINOBU MAEDA ${ }^{1}$ and KATSUYUKI KIURA ${ }^{2}$ \\ ${ }^{1}$ Department of Hematology, Oncology and Respiratory Medicine, Okayama University Graduate School of Medicine, \\ Dentistry and Pharmaceutical Sciences; ${ }^{2}$ Department of Respiratory Medicine, ${ }^{3}$ Okayama University Hospital Biobank, \\ ${ }^{4}$ Center for Innovative Clinical Medicine and ${ }^{5}$ Center for Clinical Oncology, Okayama University Hospital; \\ ${ }^{6}$ Department of General Thoracic Surgery and Breast and Endocrinological Surgery, Okayama University \\ Graduate School of Medicine, Dentistry and Pharmaceutical Sciences, Okayama 700-8558, Japan
}

Received Jun 6, 2020; Accepted September 9, 2020

DOI: $10.3892 / \mathrm{ol} .2020 .12256$

\begin{abstract}
The detection of certain oncogenic driver mutations, including those of epidermal growth factor receptor $(E G F R)$, is essential for determining treatment strategies for advanced non-small cell lung cancer (NSCLC). The current study assessed the feasibility of testing exhaled breath condensate (EBC) for EGFR mutations by droplet digital PCR (ddPCR). Samples were collected from 12 patients with NSCLC harboring EGFR mutations that were admitted to Okayama University Hospital between June 1, 2014 and December 31, 2017. A total of 21 EBC samples were collected using the RTube ${ }^{\mathrm{TM}}$ method and EGFR mutations (L858R, exon 19 deletions or T790M) were assessed through ddPCR analysis (EBC-ddPCR). A total of 3 healthy volunteer samples were also tested to determine a threshold value for each mutation. Various patient characteristics were
\end{abstract}

Correspondence to: Dr Kadoaki Ohashi, Department of Respiratory Medicine, Okayama University Hospital, 2-5-1 Shikata-cho Kita Ward, Okayama 700-8558, Japan

E-mail: kohashi@cc.okayama-u.ac.jp

Abbreviations: EGFR, epidermal growth factor receptor; PCR, polymerase chain reaction; ddPCR, droplet digital polymerase chain reaction; NSCLC, non-small cell lung cancer; EBC, exhale breath condensate; DNA, deoxyribonucleic acid; PNA, peptide nucleic acid; LNA, locked nucleic acid; TKI, tyrosine kinase inhibitor; HER2, human epidermal growth factor receptor type 2; EBC-ddPCR, ddPCR using EBC

Key words: non-small cell lung cancer, epidermal growth factor receptor mutations, droplet digital PCR, exhaled breath condensate, EGFR-TKIs determined, including sex (3 males and 9 females), age (range 54-81 years; median, 66 years), smoking history (10 had never smoked; 2 were former smokers), histology (12 patients exhibited adenocarcinoma), clinical stage ( 9 patients were stage IV; 3 exhibited post-operative recurrence) and EGFR mutation type (4 had L858R; 8 had exon 19 deletions; 8 had T790M). EBC-ddPCR demonstrated positive droplets in 8 of the 12 patients. The sensitivity and specificity of each mutation was as follows: 27.3 and $80.0 \%$ for EGFR L858R, 30.0 and $90.9 \%$ for EGFR Ex19del, and 22.2 and $100 \%$ for $E G F R$ T790M. EBC-ddPCR analysis of EGFR mutations exhibited modest sensitivity and acceptable specificity. EBC-ddPCR is a minimally invasive and replicable procedure and may be a complementary method for EGFR testing in patients where blood or tissue sampling proves difficult.

\section{Introduction}

The detection of oncogenic driver mutations, such as epidermal growth factor receptor (EGFR) mutations, is essential for determining treatment strategies for advanced non-small cell lung cancer (NSCLC). The EGFR-tyrosine kinase inhibitor (TKI) is among the most successful treatments for NSCLC with EGFR mutations, as it extends the median overall survival by over 30 months $(1,2)$. A third-generation EGFR-TKI, osimertinib, has been clinically approved for lung cancers harboring the acquired resistance mutation EGFR T790M (3). However, the invasive tissue biopsy required for $E G F R$ testing is often challenging. Indeed, data show that re-biopsy was not performed in $20-50 \%$ of patients treated with EGFR-TKIs, raising questions on the viability of this method (4-6).

Liquid biopsies that assess circulating tumor DNA from blood samples have been developed, providing an alternative for biomarker identification $(7,8)$. Other samples, such as 
Table I. Patient characteristics.

\begin{tabular}{|c|c|c|c|c|c|c|}
\hline Patient number & Sex & Age (years) & Smoking & Stage $^{b}$ & Baseline EGFR mts & Biopsy site \\
\hline 1 & $\mathrm{~F}$ & 60 & Never & rec & L858R & $-{ }^{\mathrm{a}}$ \\
\hline 2 & M & 68 & Former & IVB & Ex19del & RL lobe \\
\hline 3 & $\mathrm{~F}$ & 81 & Never & IVB & Ex19del & Pleural fluid \\
\hline 4 & $\mathrm{~F}$ & 74 & Never & IVB & Ex19del & RU lobe \\
\hline 5 & $\mathrm{~F}$ & 66 & Never & rec & Ex19del & RU lobe \\
\hline 6 & $\mathrm{~F}$ & 58 & Never & IVB & Ex19del & Pleural fluid \\
\hline 7 & M & 76 & Never & IVA & L858R & Pleura \\
\hline 8 & $\mathrm{~F}$ & 67 & Never & rec & L858R & Middle lobe \\
\hline 9 & $\mathrm{~F}$ & 70 & Former & IVB & L858R & Pleural fluid \\
\hline 10 & M & 56 & Never & IVB & Ex19del & RU lobe \\
\hline 11 & $\mathrm{~F}$ & 56 & Never & IVB & Ex19del & LL lobe \\
\hline 12 & $\mathrm{~F}$ & 54 & Never & IVB & Ex19del & RL lobe \\
\hline
\end{tabular}

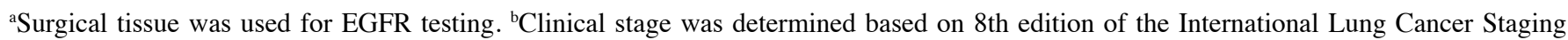
System. EGFR, epidermal growth factor receptor; F, female; M, male; rec, recurrence; Ex19del, EGFR exon 19 deletion; RL, right lower; RU, right upper; LL, left lower

Table II. Healthy volunteer characteristics.

\begin{tabular}{lccc}
\hline Healthy volunteer & Sex & Age & Smoking \\
\hline 1 & M & 41 & Never \\
2 & M & 33 & Never \\
3 & M & 37 & Never
\end{tabular}

M, male.

exhaled breath condensate (EBC) and urine, have also been tested as alternatives to liquid biopsy (9-12).

We previously reported the detection of EGFR exon 19 deletion (Ex19del) mutations in EBC by conventional polymerase chain reaction (PCR) (13). Recently, a highly sensitive droplet digital PCR (ddPCR) was developed for liquid biopsy (14-16). In this study, we investigated whether EBC testing using ddPCR (EBC-ddPCR) for EGFR mutations is viable in patients with NSCLC.

\section{Patients and methods}

Clinical samples and lung cancer cell lines. Patients with lung cancer harboring the EGFR mutations L858R, Ex19del, or T790M were enrolled in this study between June 1, 2014 and December 31, 2017 after obtaining written informed consent. All patients were diagnosed with NSCLC using surgical tissue samples, biopsy specimens, or cytology samples. The diagnosis was based on the General Rules for the Clinical and Pathological Classification of Lung Cancer of the Japan Lung Cancer Society (8th edition) and TNM staging system of the International Association for the Study of Lung Cancer (8th edition) (17). Written informed consent was also obtained from three healthy volunteers. The baseline EGFR mutations of lung cancers were confirmed by clinically approved methods in Japan by practically examining surgical tissues, biopsy specimens, or cytology samples. Approximately 1-2 ml of EBC was collected using RTube ${ }^{\mathrm{TM}}$ (Respiratory Research), according to the manufacturer's protocol. After collecting the EBC, 1-ml aliquots were dispensed and stored at $-80^{\circ} \mathrm{C}$. This study was approved by the Ethics Committee of Okayama University (Authorization number: 2221).

The lung cancer cell line H3255 harboring EGFR L858R was kindly provided by Dr William Pao (Vanderbilt University, Nashville, TN, USA) (18). The gefitinib-resistant lung cancer cell lines RPC-9 harboring EGFR Ex19del and T790M were previously established in our laboratory (19).

Droplet digital PCR assay for EGFR mutation detection. DNA was extracted using the QIAamp DNA Mini kit (Qiagen $\mathrm{GmbH}$ ) according to the manufacturer's protocol. DNA qualification was performed with a NanoDrop spectrometer (Thermo Fisher Scientific, Inc.). The following primer and probe kits were purchased from Bio-Rad): ddPCR Mutation assay: EGFR p.L858R c.2573T>G (\#10049550); ddPCR EGFR Exon 19 Deletions Screening kit (\#12002392) and ddPCR Mutation assay: EGFR p.T790M, Human (\#10049550). ddPCR was performed at Biobank (Okayama University Hospital, Okayama, Japan) using the QX200 Droplet Digital PCR system (Bio-Rad) according to the manufacturer's protocol. The following conditions were used for ddPCR: i) An initial denaturation step at $95^{\circ} \mathrm{C}$ for $10 \mathrm{~min}$ followed by: ii) 45 cycles at $94^{\circ} \mathrm{C}$ for $30 \mathrm{sec}$; and iii) 45 cycles at ${ }^{\circ} \mathrm{C}$ for $1 \mathrm{~min}$, with a 4) final enzyme deactivation step at $98^{\circ} \mathrm{C}$ for $10 \mathrm{~min}$. The ramp rate for all steps was $2^{\circ} \mathrm{C} / \mathrm{sec}$. PCR products were then subjected to analysis with a QX-200 Droplet reader and QuantaSoft analysis software (Bio-Rad), with a EGFR Specific Multiplex DNA Reference Standard (\#HD802, Horizon Discovery) used as a positive control. The accuracy of the ddPCR was confirmed using serially diluted DNA of lung cancer cell lines (Fig. S1A-F). The patient samples and control samples including EBC samples from three healthy-volunteers 


\section{Baseline Patient mutation No}

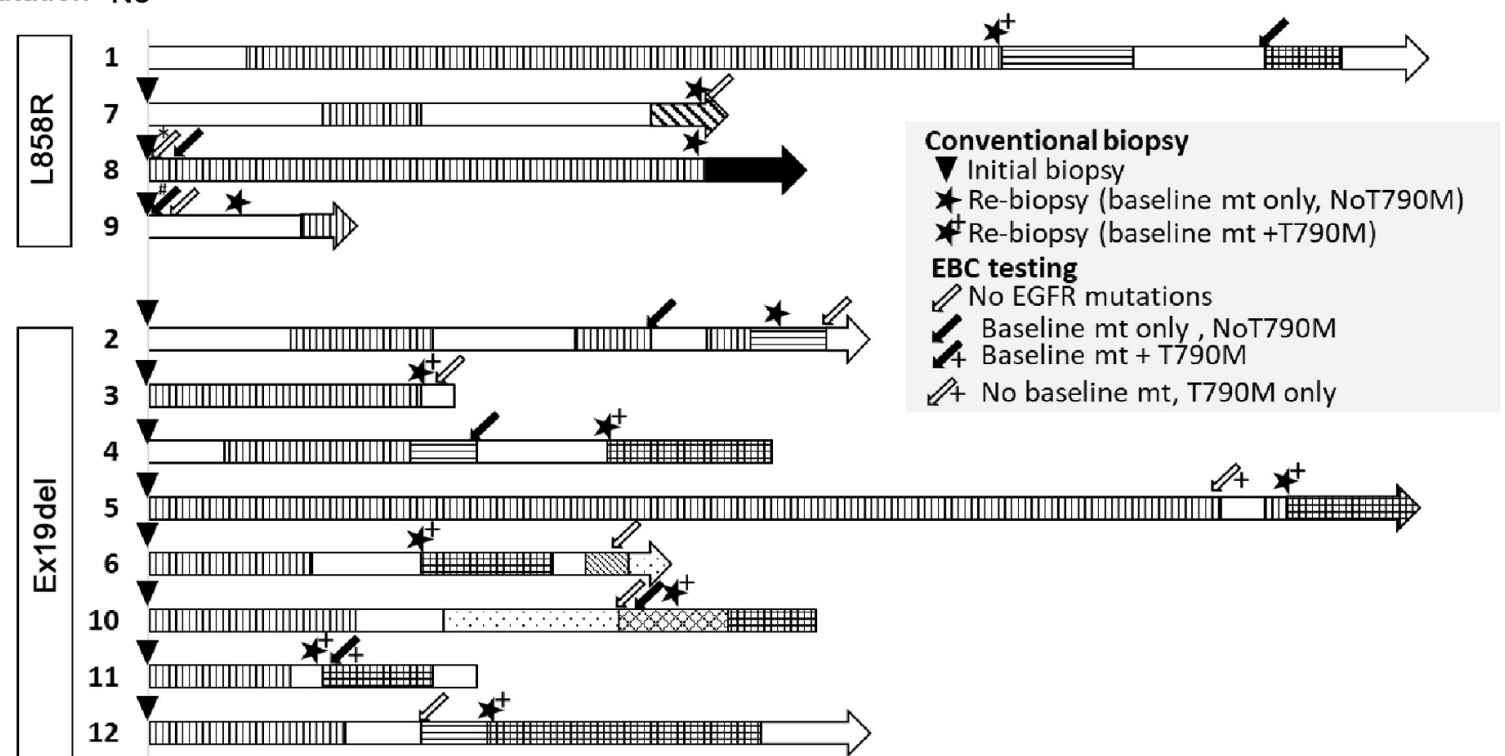

0

20

40

60

80

100

120 Months

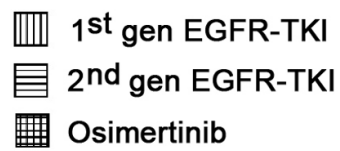

Investigational $3^{\text {rd }}$ gen EGFR-TKI

Investigational HER2 inhibitor

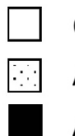

Chemotherapy

Anti-PD-1 ab

Anti-PD-L1 ab + chemotherapy

Figure 1. Flow diagram of the clinical course of 12 patients with lung cancer harboring EGFR mutations and the time of biopsy or exhaled breath condensate sampling. "Three consecutive samples were collected. ${ }^{\#}$ Four consecutive samples were collected. EGFR, epidermal growth factor receptor; TKI, tyrosine kinase inhibitor; Ex19del, EGFR exon 19 deletions; Anti-PD-1 ab, anti-programmed cell death-1 antibody; Anti-PD-L1 ab, anti-programmed cell death ligand-1 antibody.

or elution buffer AE from the QIAamp DNA Mini kit (Qiagen) as negative controls were tested to determine the threshold (Fig. S2A-D). No droplets were detected for EGFR L858R or $E G F R$ T790M, whereas positive reactions were observed for EGFR Ex19del in the negative control samples. Therefore, the threshold for positive results for EGFR Ex19del were analyzed with the receiver operating characteristic (ROC) using patient samples (Fig. S3). As a result, the threshold for $E G F R$ mutation-positive was defined as follows: $E G F R$ L858R ( $\geq 0.01$ copies/ $/ \mu$ ), EGFR Ex19del ( $\geq 0.5$ copies/ $\mu 1$ ), and $E G F R$ T790M ( $\geq 0.01$ copies/ $\mu \mathrm{l})$.

Statistical analysis. Statistical analysis was performed using STATA software version 15.1 (StataCorp). ROC analysis was performed to determine the optimal threshold for epidermal growth factor receptor exon 19 deletion. The $95 \%$ confidence interval (CI) was calculated by using the Clopper-Pearson exact method for binomial proportions.

\section{Results}

Patient characteristics and EBC samples. Patient and healthy control characteristics are detailed in Tables I and II, respectively. The median patient age was 66 years (range, 54-81), comprising 3 males and 9 females: 10/12 were never-smokers and 9/12 were in stage IV of the disease. Four lung tumors harbored EGFR L858R, whereas 8 tumors showed the EGFR Ex19del mutation (Table I). In total, 21 samples were collected from the 12 patients. Of these 21 samples, 11 were from patients with lung cancers harboring $E G F R$ L858R and 10 were from patients with lung cancers harboring $E G F R$ Ex19del. The timing of EBC collection and treatment history are shown in Fig. 1.

Nine EBC samples were collected from two patients at the time of initiation of EGFR-TKI (Table III). In contrast, 12 EBC samples were collected from ten patients at the time of the second biopsy (Table IV). There were no adverse events due to EBC sampling.

EBC-ddPCR for EGFR mutations in patients. The $21 \mathrm{EBC}$ samples from 12 patients were analyzed by ddPCR using $E G F R$ L858R, Ex19del. or T790M primer sets. In the four patients with lung cancer harboring EGFR L858R, 3 of 11 EBC samples (sample nos. 1-1, 8-4, and 9-4) were positive for EGFR L858R (Fig. 2A and Tables II and IV). In the 8 patients with lung cancer harboring EGFR Ex19del, 3/10 EBC samples (sample nos. 2-1, 4-1, and 11-1) were positive for EGFR Ex19del (Fig. 2B and Table IV). In the 8 patients with lung cancer harboring EGFR T790M, 2/9 EBC samples (sample nos. 5-1 and 11-1) were positive for EGFR T790M (Fig. 2C and Table IV). No association was detected between the detection of mutations and T-factor/tumor localization in the lung or quality/quantity of DNA in the EBC samples (Tables III and IV).

Consequently, the sensitivity of the EBC test for EGFR mutations was as follows: $27.3 \%$ (95\% CI, 6.0-61.0\%) for EGFR L858R, 30.0\% (95\% CI, 6.7-65.2\%) for EGFR Ex19del, and $22.2 \%$ (95\% CI, 2.8-60.0\%) for EGFR T790M. The specificity 
Table III. EBC-ddPCR.

\begin{tabular}{|c|c|c|c|c|c|c|c|c|}
\hline Patient no. & Baseline $E G F R \mathrm{mts}$ & Biopsy site & T-factor & $\begin{array}{l}\text { Localization } \\
\text { of lung tumors }\end{array}$ & Sample no. & DNA (ng) & $\begin{array}{l}260 / 280 \\
\text { ratio }\end{array}$ & EBC-ddPCR \\
\hline \multirow[t]{4}{*}{8} & L858R & RL lobe & $2 b$ & Peri & $8-1$ & 279.5 & 1.46 & $(-)$ \\
\hline & & & & & $8-2$ & 133.5 & 1.37 & $(-)$ \\
\hline & & & & & $8-3$ & 503.0 & 1.73 & $(-)$ \\
\hline & & & & & $8-4$ & 250.0 & 1.86 & L858R \\
\hline \multirow[t]{5}{*}{9} & L858R & Pleural fluid & 4 & Center & $9-1$ & 138.0 & 1.66 & $(-)$ \\
\hline & & & & & $9-2$ & 257.0 & 1.53 & $(-)$ \\
\hline & & & & & $9-3$ & 253.5 & 2.06 & $(-)$ \\
\hline & & & & & $9-4$ & 267 & 1.99 & L858R \\
\hline & & & & & $9-5$ & 181.5 & 1.37 & $(-)$ \\
\hline
\end{tabular}

EBC, exhaled breath concentrate; ddPCR, droplet digital PCR; EGFR, epidermal growth factor receptor; mts, mutations; T-factor, T-factor of TNM staging system version 8; RL, right lower.

\section{A Copy number of L858R}

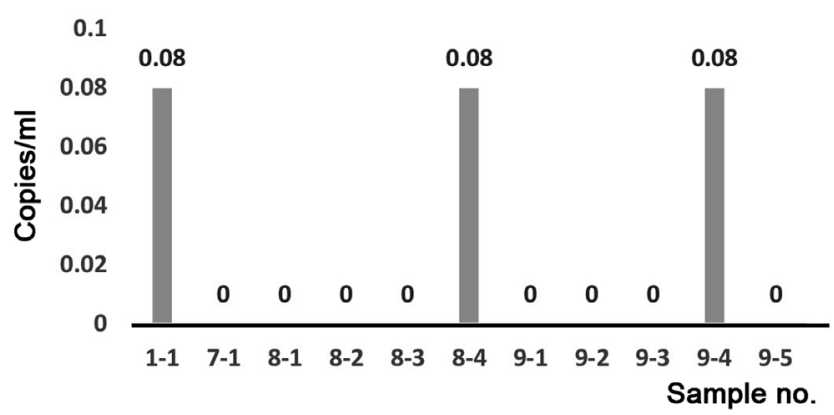

B Copy number of Ex19del
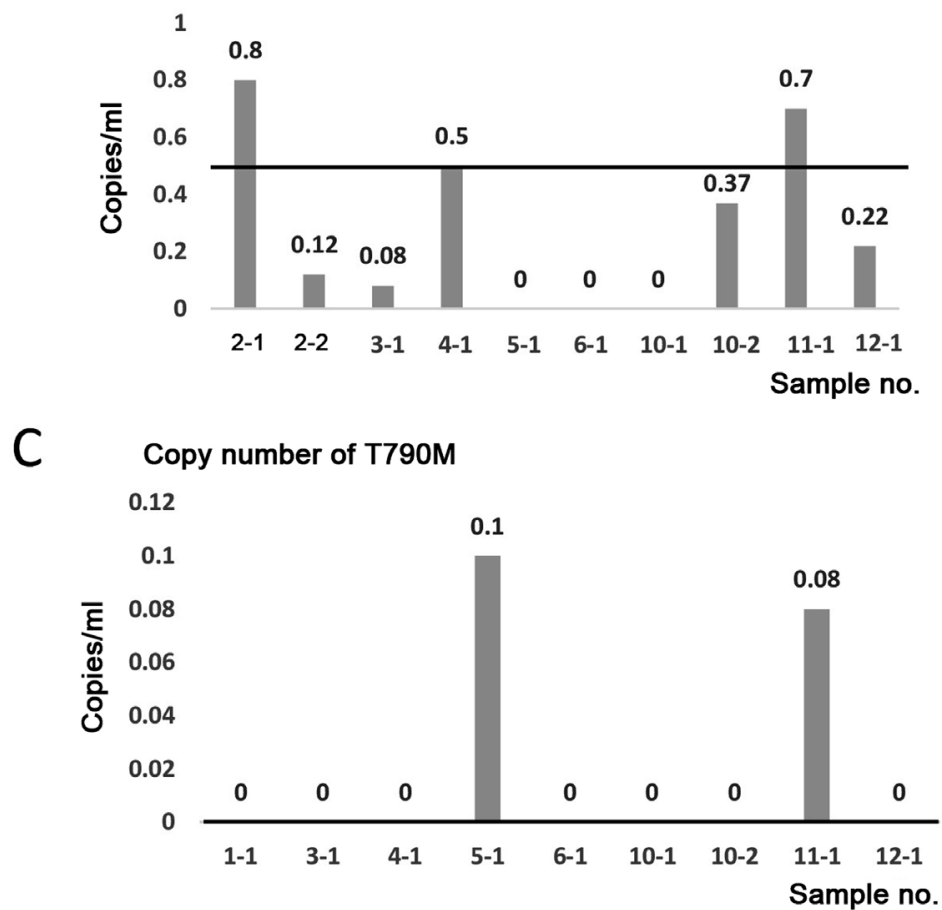

Figure 2. Detection of EGFR mutations by EBC-ddPCRA. (A) ddPCR was performed using EGFR L858R primer sets. EGFR L858R was amplified in EBC samples 1-1, 8-4 and 9-4. (B) ddPCR was performed using EGFR exon 19 deletion primer sets. EGFR exon 19 deletions were amplified in EBC samples 2-1, 4-1 and 11-1. (C) ddPCR was performed using EGFR T790M primer sets. EGFR T790M was amplified in EBC samples 5-1 and 11-1. The black bars indicate the positive threshold, which was 0 copies $/ \mu$ l for $E G F R$ L858R and $E G F R$ T790M, and 0.5 copies $/ \mu 1$ for $E G F R$ exon 19 deletion. EGFR, epidermal growth factor receptor; EBC, exhaled breath condensate; ddPCR, droplet digital PCR; Ex19del, EGFR exon 19 deletions. 
Table IV. EBC-ddPCR.

\begin{tabular}{|c|c|c|c|c|c|c|c|c|c|}
\hline $\begin{array}{l}\text { Patient } \\
\text { no. }\end{array}$ & $\begin{array}{c}\text { 2nd-biopsy } \\
E G F R \text { mutations }\end{array}$ & $\begin{array}{l}\text { EGFR } \\
\text { test }\end{array}$ & Biopsy site & T-factor & $\begin{array}{l}\text { Localization } \\
\text { of lung tumors }\end{array}$ & $\begin{array}{l}\text { Sample } \\
\text { no. }\end{array}$ & DNA (ng) & $\begin{array}{c}260 / 280 \\
\text { ratio }\end{array}$ & EBC-ddPCR \\
\hline 1 & L858R + T790M & Clamp & LL lobe & $\mathrm{Tx}^{\mathrm{a}}$ & Peri & $1-1$ & 214.5 & 1.37 & L858R \\
\hline \multirow[t]{2}{*}{2} & Ex19del & Clamp & RL lobe & 4 & Center & $2-1$ & 268.5 & 2.33 & Ex19del \\
\hline & & & & & & $2-2$ & 234.0 & 1.88 & $(-)$ \\
\hline 3 & Ex19del + T790M & Clamp & Pleural fluid & $2 \mathrm{a}$ & Peri & $3-1$ & 272.0 & 192.0 & $(-)$ \\
\hline 4 & Ex19del + T790M & TaqMan & Blood & $2 \mathrm{a}$ & Peri & $4-1$ & 244.5 & 2.17 & Ex19del \\
\hline 5 & Ex19del + T790M & Clamp & CSF & $\mathrm{Tx}^{\mathrm{b}}$ & Peri & $5-1$ & 277.0 & 1.64 & T790M \\
\hline 6 & Ex19del + T790M & Clamp & Pleural fluid & $1 b$ & Peri & $6-1$ & 280.5 & 2.07 & $(-)$ \\
\hline 7 & L858R & Clamp & Pleural fluid & $2 \mathrm{a}$ & Peri & $7-1$ & 420.5 & 1.55 & $(-)$ \\
\hline \multirow[t]{2}{*}{10} & Ex19del + T790M & Clamp & Pleural fluid & $1 b$ & Peri & $10-1$ & 123.5 & 10.48 & $(-)$ \\
\hline & & & & & & $10-2$ & 163.5 & 4.41 & $(-)$ \\
\hline 11 & Ex19del + T790M & Clamp & LL lobe & 3 & Peri & $11-1$ & 152.0 & 4.01 & Ex19del + T790M \\
\hline 12 & Ex19del + T790M & Clamp & RU lobe & $2 \mathrm{a}$ & Peri & $12-1$ & 323.0 & 1.66 & $(-)$ \\
\hline
\end{tabular}

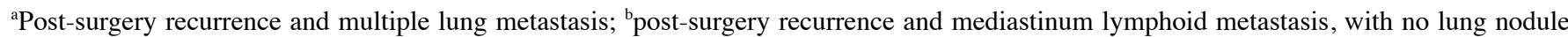
observed. EBC, exhaled breath concentrate; ddPCR, droplet digital PCR; EGFR, epidermal growth factor receptor; T-factor, T-factor of TNM staging system version 8; Clamp, the peptide nucleic acid-locked nucleic acid PCR clamp; TaqMan, TaqMan assay; Ex19del, EGFR exon 19 deletion; LL, left lower; peri, peripheral; RL, right lower; RU, right upper; CSF, cerebrospinal fluid.

Table V. Sensitivity, specificity and predictive values of exhaled breath concentrate-droplet digital PCR.

\begin{tabular}{lllr}
\hline Parameter & L858R & Ex19del & T790M \\
\hline Sensitivity $(95 \% \mathrm{CI})$ & $27.3(6.0-61.0)$ & $30.0(6.7-65.2)$ & $22.2(2.8-60.0)$ \\
Specificity $(95 \% \mathrm{CI})$ & $80.0(44.4-97.5)$ & $90.9(58.7-99.8)$ & $100(73.5-100)$ \\
Positive predictive value $(95 \% \mathrm{CI})$ & $60.0(14.7-94.7)$ & $75.0(19.4-99.4)$ & $100(15.8-100)$ \\
Negative predictive value $(95 \% \mathrm{CI})$ & $50.0(24.7-75.3)$ & $58.8(32.9-81.6)$ & $63.2(38.4-83.7)$ \\
\hline
\end{tabular}

95\% CIs were calculated using the Clopper-Pearson exact method for binomial proportions. Ex19del, EGFR exon 19 deletion; CI, confidence interval

was as follows: $80.0 \%$ (95\% CI, 44.4-97.7\%) for EGFR L858R, 90.9\% (95\% CI, 58.7-99.8\%) for EGFR Ex19del, and $100 \%$ (95\% CI, 73.5-100\%) for EGFR T790M (Table V).

Clinical course of highlighted cases with lung adenocarcinoma, whose EGFR mutations were detected by EBC-ddPCR Patient number 8. A non-smoking 67-year-old woman was diagnosed with synchronous double primary lung cancer composed of adenocarcinoma harboring EGFR L858R in the right lower lobe of the lung, and adenocarcinoma harboring EGFR Ex19del in the right upper lobe of the lung. A right lower lobectomy (pathological stage T2bN0M0 cStage IIA) and right segment 3 a partial resection (pathological stage T1aN0M0 cStage IA1) were performed, with four subsequent courses of adjuvant chemotherapy with a combination of cisplatin and vinorelbine. However, at 12 months post-surgery, a new lesion appeared in the middle lobe. Transbronchial biopsy of the lesion revealed an adenocarcinoma harboring EGFR L858R. The patient's Eastern Cooperative Group Performance Status was grade 1, and thus gefitinib was administered at $250 \mathrm{mg}$ daily. EBC was collected 1 day prior starting gefitinib and at 2, 16 and 62 days after gefitinib initiation. Gefitinib was discontinued from days 41-62 because of liver damage, but later re-administered at a reduced dosage of $250 \mathrm{mg}$ every 2 days. EBC-ddPCR detected EGFR L858R only in the fourth sampling (21 days after gefitinib cessation). The maximum therapeutic effect of gefitinib was a partial response (Response Evaluation Criteria in Solid Tumors version 1.1), with a progression-free survival of 48 months (Fig. 3A and B).

Patient number 11. A non-smoking 56-year-old woman was diagnosed with adenocarcinoma in the left lower lobe of the lung (clinical stage T3N1M1b cStage IVB, multiple brain metastases, bone metastases). The patient's Eastern Cooperative Group Performance Status was grade 1. EGFR Ex19del was detected in the biopsied tissue, after which erlotinib was administered. However, at 12 months after initiating erlotinib, the primary tumor increased. Two subsequent cycles of cytotoxic chemotherapy with cisplatin and pemetrexed were administered, but the tumor regrew following treatment. Re-biopsy was performed on primary lung tumor and EBC was collected at the same time. In addition to the baseline EGFR Ex19del mutation, EGFR T790M was also detected in the biopsy sample. Similarly, EGFR Ex19del and T790M 
A

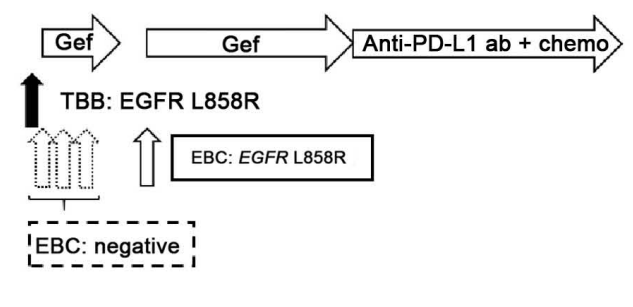

B

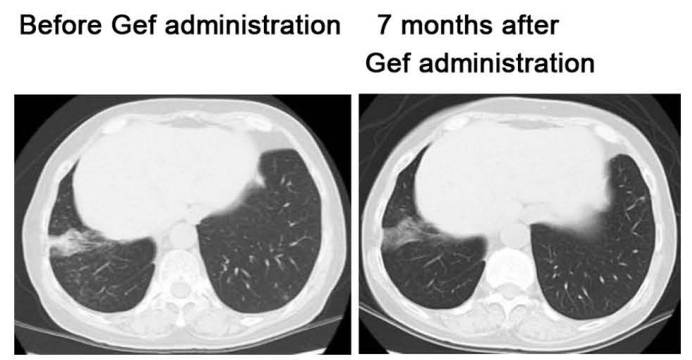

C

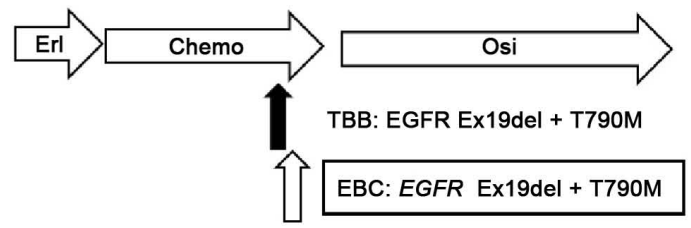

D

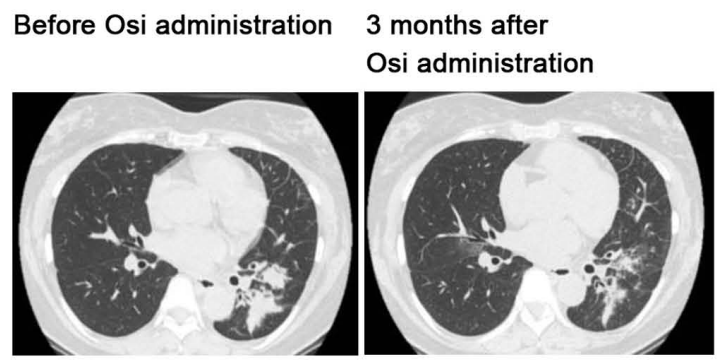

Figure 3. Clinical course of highlighted cases. (A) Clinical course of patient 8. (B) Chest CT image obtained 7 months after the initiation of Gef treatment. A lesion reduction in the right lower lobe can be observed. (C) Clinical course of patient 11. (D) Chest CT image obtained 3 months after the initiation of Osi treatment. Lesion reduction in the left lower lobe is exhibited. Erl, Erlotinib; Chemo, cytotoxic chemotherapy; TBB, transbronchial biopsy; EBC, exhaled breath condensate; Gef, gefitinib; Osi, osimertinib; Anti-PD-L1 ab, anti-programmed cell death ligand 1 antibody; Ex19del, EGFR exon 19 deletions. EGFR, epidermal growth factor receptor.

were detected by the EBC-ddPCR method. Subsequently osimertinib was administered and continued over 6 months, which caused a partial response (Response Evaluation Criteria in Solid Tumors version 1.1) (Fig. 3C and D).

\section{Discussion}

This study demonstrated that using EBC-ddPCR to detect EGFR mutations is feasible and shows a modest sensitivity (20-30\%) and acceptable sensitivity (80-100\%) in patients with lung cancers harboring EGFR mutations.

Up to $60 \%$ of lung adenocarcinomas treated with first- or second-generation EGFR-TKIs develop resistant EGFR T790M mutations $(20,21)$. However, third-generation EGFR-TKIs were only administered in $23.7 \%$ of patients (22). A negative result for EGFR T790M in re-biopsied samples should not prevent physicians from performing a repeat biopsy; however, it is not always possible to carry out repeat biopsies $(21,23)$. The sensitivity of EBC testing for EGFR mutations was modest compared to that of blood tests (8); however, EBC testing is much more easily repeated because of its minimal invasiveness. In this study, we performed three or four repeated EBC samplings without any adverse effects in both case 8 and 9 . Although we did not assess the concordance between tissue biopsy and EBC testing on a larger scale, gefitinib and osimertinib inhibited the lung tumor in cases 8 and 11, respectively, with both the tissue biopsy and EBC testing detecting EGFR L858R or EGFR Ex19del and T790M. These cases suggest the potential of EBC testing as a complementary option for patients in whom tissue biopsy is difficult or for those who refuse repeated blood sampling.

Although ddPCR is thought to detect $0.005-0.1 \%$ of target DNA (24), this study revealed a modest sensitivity for EGFR mutations in EBC samples (20-30\%). Smyth et al reported that EGFR T790M was detected in 9/10 EBC samples by UltraSEEK ${ }^{\mathrm{TM}}$ technology (12). Possible explanations for the discrepancy between the previous report and our study are differences in sample quality and sensitivity of the detection methods.

This study had several limitations. The sample size was small ( $\mathrm{n}=21$ in 12 patients) and the number of EBC samples from treatment naïve patients and samples prior to initiating EGFR-TKI were from only 1 case; therefore, patient bias should be considered. Furthermore, concordance among EBC testing, blood testing, and tissue biopsy was not assessed in detail. Therefore, these data should be considered as exploratory.

EBC-ddPCR for EGFR mutations by ddPCR was feasible and showed moderate sensitivity and acceptable specificity. EBC sampling is minimally invasive and replicable; therefore, EBC tests could be a complementary option for patients in whom tissue biopsy is difficult or for those who refuse repeated blood sampling. Further studies are needed to explore the potential of the EBC test.

\section{Acknowledgements}

The authors would like to thank Mrs. Hiromi Nakashima (Okayama University Graduate School of Medicine, Dentistry and Pharmaceutical Sciences) and Mrs. Kyoko Maeda (Okayama University Graduate School of Medicine, Dentistry and Pharmaceutical Sciences) for providing technical support.

\section{Funding}

The present study was supported by JSPS for Scientific Research Grant-in-Aid for Young Scientists (B): KAKEN (grant no. 16K19454). The current research was also supported by 
Chugoku Occupational Health Association (grant no. CRE 17-2) and Okayama health foundation (grant no. 33K-2017-03).

\section{Availability of data and materials}

The datasets used and/or analyzed during the current study are available from the corresponding author on reasonable request.

\section{Authors' contributions}

Data interpretation and presentation were the sole responsibility of the authors. KaN and $\mathrm{KO}$ had full access to all data and assume responsibility for data integrity and the accuracy of data analysis. KaN and $\mathrm{KO}$ contributed to the study design, data collection, analyses, and manuscript writing. TT, KiN, TM, SS, HK, HW, NO, GM, HH, YK, TN, TK, HY, STom, KH, MT, SToy, YM, KK collected the data and prepared the manuscript. All authors read and approved the final manuscript.

\section{Ethics approval and consent to participate}

The present study was approved by the Institute Research Ethics Committee of the Okayama University Hospital and written informed consent was obtained from all patients.

\section{Patient consent for publication}

Written informed consent regarding publication was obtained from all patients.

\section{Competing interests}

Dr Kadoaki Ohashi reports research funding from Boehringer Ingelheim, Novartis, AstraZeneca, Eli Lilly, MSD, and Daiichi-Sankyo outside the submitted work. Dr Kadoaki Ohashi reports personal fees from AstraZeneca, MSD, and Chugai Pharmaceutical outside the submitted work.

\section{References}

1. Kris MG, Johnson BE, Berry LD, Kwiatkowski DJ, Iafrate AJ, Wistuba II, Varella-Garcia M, Franklin WA, Aronson SL, Su PF, et al: Using multiplexed assays of oncogenic drivers in lung cancers to select targeted drugs. JAMA 311: 1998-2006, 2014

2. Okamoto I, Morita S, Tashiro N, Imamura F, Inoue A, Seto T, Yamamoto N, Ohe Y, Nakagawa K and Fukuoka M: Real-world treatment and outcomes in EGFR mutation-positive non-small cell lung cancer: Long-term follow-up of a large patient cohort. Lung Cancer 117: 14-19, 2018.

3. MokTS,Wu Y-L,AhnM-J,GarassinoMC,KimHR,RamalingamSS, Shepherd FA, He Y, Akamatsu H, Theelen WS, et al: Osimertinib or platinum-pemetrexed in EGFR T790M-positive lung cancer. N Engl J Med 376: 629-640, 2017.

4. Nosaki K, Satouchi M, Kurata T, Yoshida T, Okamoto I, Katakami N, Imamura F, Tanaka K, Yamane Y, Yamamoto N, et al Re-biopsy status among non-small cell lung cancer patients in Japan: A retrospective study. Lung Cancer 101: 1-8, 2016.

5. Chouaid C, Dujon C, Do P, Monnet I, Madroszyk A, Le Caer H, Auliac JB, Berard H, Thomas P, Lena H, et al: Feasibility and clinical impact of re-biopsy in advanced non-small-cell lung cancer: A prospective multicenter study in a real-world setting (GFPC study 12-01). Lung Cancer 86: 170-173, 2014.

6. Kim TO, Oh IJ, Kho BG, Park HY, Chang JS, Park CK, Shin HJ, Lim JH, Kwon YS, Kim YI, et al: Feasibility of re-biopsy and EGFR mutation analysis in patients with non-small cell lung cancer. Thorac Cancer 9: 856-864, 2018.
7. Crowley E, Di Nicolantonio F, Loupakis F and Bardelli A: Liquid biopsy: Monitoring cancer-genetics in the blood. Nat Rev Clin Oncol 10: 472-484, 2013.

8. Oxnard GR, Thress KS, Alden RS, Lawrance R, Paweletz CP, Cantarini M, Yang JC, Barrett JC and Jänne PA: Association between plasma genotyping and outcomes of treatment with osimertinib (AZD9291) in advanced non-small-cell lung cancer. J Clin Oncol 34: 3375-3382, 2016.

9. Husain H, Melnikova VO, Kosco K, Woodward B, More S, Pingle SC, Weihe E, Park BH, Tewari M, Erlander MG, et al: Monitoring daily dynamics of early tumor response to targeted therapy by detecting circulating tumor DNA in urine. Clin Cancer Res 23: 4716-4723, 2017.

10. Carpagnano GE, Foschino-Barbaro MP, Mulé G, Resta O, Tommasi S, Mangia A, Carpagnano F, Stea G, Susca A, Di Gioia G, et al: 3P microsatellite alterations in exhaled breath condensate from patients with non-small cell lung cancer. Am J Respir Crit Care Med 172: 738-744, 2005.

11. Youssef O, Knuuttila A, Piirilä P, Böhling T, Sarhadi V and Knuutila S: Hotspot mutations detectable by next-generation sequencing in exhaled breath condensates from patients with lung cancer. Anticancer Res 38: 5627-5634, 2018.

12. Smyth RJ, Toomey SM, Sartori A, O'Hanrahan E, Cuffe SD, Breathnach OS, Morgan RK and Hennessy BT: Brief report on the detection of the EGFR T790M mutation in exhaled breath condensate from lung cancer patients. J Thorac Oncol 13: 1213-1216, 2018.

13. Zhang D, Takigawa N, Ochi N, Tanimoto Y, Noujima D, Chen YY, Tanimoto $M$ and Kiura K: Detection of the EGFR mutation in exhaled breath condensate from a heavy smoker with squamous cell carcinoma of the lung. Lung Cancer 73: 379-380, 2011.

14. Sacher AG, Paweletz C, Dahlberg SE, Alden RS, O'Connell A, Feeney N, Mach SL, Jänne PA and Oxnard GR: Prospective validation of rapid plasma genotyping for the detection of EGFR and KRAS mutations in advanced lung cancer. JAMA Oncol 2: 1014-1022, 2016.

15. Suzawa K, Yamamoto H, Ohashi K, Hashida S, Tomida S, Kubo T, Maki Y, Soh J, Tsukuda K, Kiura K, et al: Optimal method for quantitative detection of plasma EGFR T790M mutation using droplet digital PCR system. Oncol Rep 37: 3100-3106, 2017.

16. Senoo S, Ohashi K, Nishii K, Hara N, Kano H, Ninomiya K, Maeda Y and Kiura K: Osimertinib depletes EGFR T790M in the spinal fluid of patients with carcinomatous meningitis of lung adenocarcinoma harboring de novo EGFR T790M. J Thorac Oncol 13: e140-e142, 2018.

17. Goldstraw P, Chansky K, Crowley J, Rami-Porta R, Asamura H, Eberhardt WE, Nicholson AG, Groome P, Mitchell A, Bolejack $\mathrm{V}$, et al: The IASLC lung cancer staging project: Proposals for revision of the TNM stage groupings in the forthcoming (eighth) edition of the TNM Classification for lung cancer. J Thorac Oncol 11: 39-51, 2016.

18. Ohashi K, Sequist LV, Arcila ME, Moran T, Chmielecki J, Lin YL, Pan Y, Wang L, de Stanchina E, Shien K, et al: Lung cancers with acquired resistance to EGFR inhibitors occasionally harbor BRAF gene mutations but lack mutations in KRAS, NRAS, or MEK1. Proc Natl Acad Sci USA 109: E2127-E2133, 2012.

19. Ogino A, Kitao H, Hirano S, Uchida A, Ishiai M, Kozuki T, Takigawa N, Takata M, Kiura K and Tanimoto M: Emergence of epidermal growth factor receptor T790M mutation during chronic exposure to gefitinib in a non-small cell lung cancer cell line. Cancer Res 67: 7807-7814, 2007.

20. Ohashi K, Maruvka YE, Michor F and Pao W: Epidermal growth factor receptor tyrosine kinase inhibitor-resistant disease. J Clin Oncol 31: 1070-1080, 2013.

21. Lee K, Kim Y, Jung HA, Lee SH, Ahn JS, Ahn MJ, Park K, Choi YL and Sun JM: Repeat biopsy procedures and T790M rates after afatinib, gefitinib, or erlotinib therapy in patients with lung cancer. Lung Cancer 130: 87-92, 2019.

22. Seto T, Nogami N, Yamamoto N, Atagi S, Tashiro N, Yoshimura Y, Yabuki Y and Saka H: Real-world EGFR T790M testing in advanced non-small-cell lung Cancer: A prospective observational study in Japan. Oncol Ther 6: 203-215, 2018.

23. Ichihara E, Hotta K, Kubo T, Higashionna T, Ninomiya K, Ohashi K, Tabata M, Maeda Y and Kiura K: Clinical significance of repeat rebiopsy in detecting the EGFR T790M secondary mutation in patients with non-small cell lung cancer. Oncotarget 9: 29525-29531, 2018.

24. Vogelstein B and Kinzler KW: Digital PCR. Proc Natl Acad Sci USA 96: 9236-9241, 1999. 